\title{
Models of Stents - Comparison and Applications
}

\author{
J. ZÁHORA, A. BEZROUK, J. HANUŠ \\ Department of Medical Biophysics, Medical Faculty in Hradec Králové, Charles University, Czech \\ Republic
}

Received May 23, 2007

Accepted May 29, 2007

On-line available May 31, 2007

\begin{abstract}
Summary
The aim of this study was to analyze the possibilities of various types of stent modeling and to develop some new models. A brief survey of basic properties of stents and a list of basic designs of stents is presented. Two approaches to stent modeling were identified. Structural mechanics is the theoretical background of our analytical model of a spiral stent. The finite element method was also used. The measurement equipment for model evaluation was developed.
\end{abstract}

\section{Key words}

Stent $\bullet$ Structural model $\bullet$ Mechanical stress $\bullet$ Finite element method

\section{Introduction}

Stents are usually defined as small tubular structures that are inserted into the diseased region via a catheter (Holzapfel et al. 2006). The main function of stents is to provide mechanical support of damaged artery or some other hollow organ to restore lumen and blood flow conditions in arteries. Recently developed stents may accumulate functions; for example, they can be used for drug delivery or for treatment of bleeding esophageal varices (removable Danis stent). Clinical failures (crushing, removing, restenosis) are some of the most serious reasons for continuing stent improvements.

As to the arteries stents are used in the case of stenosis, aneurysm or dissection. The most frequent usage of stents is to prop open the constricted lumen of the atherosclerotic artery. Typically they are applied to the coronary, brain, renal, thoracic, abdominal and peripheral arteries. But they are used also for treatment of obstructions in the urethral, biliary and gastrointestinal (colorectal stents) tract, in trachea and larynx. And some special applications may be found, such as the vaginal or esophageal stent as mentioned above.

Models are an indispensable part of further stent improvement and development. They take into account both material and geometrical parameters. As a result of such models both type parameters (displacements, reaction forces, radial stiffness, bending flexibility, stresses, strains, extent of permanent deformation, elastic spring-back) or their visualization (static, dynamic, interaction with artery wall) may be considered. The new design should be tested experimentally. But it is very often expensive and time-consuming, especially due to the fatigue life. For example, if the stent has a 10-year fatigue life and the heart rate is 75 beats per min, it corresponds to approximately 400 million cyclic pulsating loadings. It may be time- and money-saving to start with modeling and simulation. It should give the designer a more detailed insight and indicate possible critical details. 


\section{Methods}

As the first step a general list of stent properties was created and the parameters that will be investigated were identified. An analysis of stent types was performed. Then the available techniques for modeling were evaluated. The resulting models were compared with one another and with experimental data. For this reason several experimental devices were developed.

\section{Properties of stents}

Only the parameters describing the stent itself were analyzed. Radial resistive force is generated by the stent to resist the reduction in its diameter; chronic outward force is the force generated by the stent when it is self-expanded toward a larger diameter (Duerig et al. 2003). Delivery size is the minimum diameter of a stent to which it can be compressed for delivering via a catheter. Crushing pressure is the critical outside pressure, which cannot be exceeded to avoid stent buckling. Shortening or expansion is very important during the pushing of a stent into or out of the catheter. Surface area is important for drug delivery. The other properties may be stiffness, fatigue life, radioopacity.

\section{Stent design overview}

At present more than 100 types of stents are available on the market (Stoeckel 2002). In some surveys they are classified in accordance with their clinical use (vascular or nonvascular, coronary or peripheral). From the more technical point of view the stents may be differentiated by material, form of material, and design. The two main groups are balloon-expandable and selfexpanding stents. The balloon-expandable stents are manufactured with a smaller diameter and, after delivery, they are dilated with a balloon. Thus the material used must be able to be plastically deformed and to remain in the expanded shape. It means it should have a low yield stress. On the other hand, self-expanding stents are manufactured so that their rest diameter is greater than the inner diameter of the target structure. The stent is constrained in a delivery system and then released and self-expanded. The material used should have in the ideal case a low elastic modulus and a high yield stress.

As to the material form, three forms are used: sheet, wire, tubing. For final mechanical properties the geometry is essential. According to Stoeckel et al. (2002) five basic types can be recognized: coil, helical spring, woven stents (braided, knitted), rings (individual, sequential), cell (closed, opened).

\section{Modeling techniques}

Two basic general approaches were determined. The basis of the first one is the structural mechanics, especially the Bernoulli-Euler beam theory and the Coulomb torsion theory. These laws may be, in a relatively easy way, applied to some simple wire structures (Wang and Ravi-Chandar 2004), e.g. some woven wire stents. The second approach is to use numerical methods. The most popular one is the finiteelements method (FEM). Its importance is growing, because more powerful computers are becoming available and a set of sophisticated software is on the market.

In an ideal cease, the problem model should be complex. It should work with the material of a stent including nonlinearities, geometrical properties of a stent, properties of surrounding tissue and interaction between a stent and the tissue, dynamics of blood flow, fatigue life. We have simplified the problem for our purpose. One region of interest is a small deformation of a stent in the artery and during artery deformation. The second one is a large deformation when a stent is pushed from the catheter.

The main output of our models should be prediction of stress-strain curves for radial and axial loading and of the diameter-length curve.

\section{Results}

\section{Equipment for measurement}

We developed several devices with two basic arrangements (Bezrouk et al. 2005, Hanus and Zahora 2005, Zahora and Hanus 2006) for experimental verification of our models. In one arrangement it is possible to measure the pressure inside and outside the covered stent which is placed in water and its diameter is measured with a camera. The second arrangement allows us to load a stent in the axial direction and to measure its elongation, force and diameter. In both arrangements measurement runs automatically. Measurement, data acquisition, and processing are controlled by our original software developed by LabVIEW, v. 7.0. For axial loading the high-precision universal material-testing device Instron 3340 was used. And also in both arrangements temperature is set and recorded.

\section{Analytical model}

We will describe how to derive all the important 


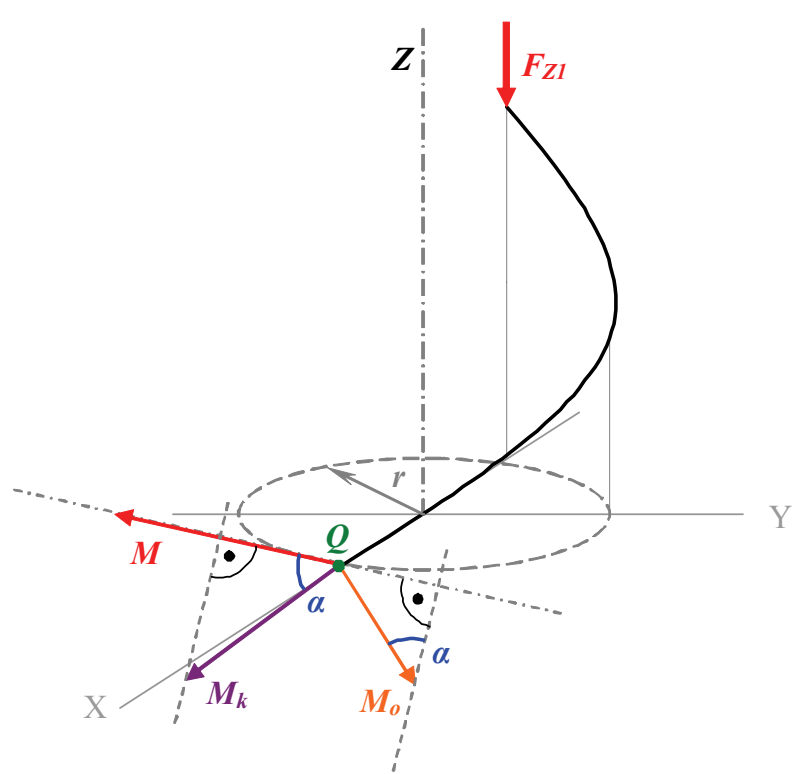

Fig. 1. Spiral stent with atraumatic edges (a) in the original shape; under a load (b) shortened; (c) elongated.

equations of the physical model of the spiral stent. First we need to find out the relationships among the basic geometrical parameters of the stent as they are, $L$ the total length, $D$ diameter, $r$ radius, $q$ pitch, $\alpha$ lead angle of a helix, $l$ the length of the wire of single turn of a helix, $n$ number of helixes, $k$ number of turns of a helix in the total length of the spiral stent. Finally we need to find out the relationships among all the acting forces and the deformation of the geometrical structure of the spiral stent. To simplify the final equations, we will define the following parameters:

Let the lead angle of a helix $\alpha$ be defined as a basic quantity describing the deformation of the spiral stent.

Let the single turn of a helix be defined as a basic element of the geometrical structure of the spiral stent hereinafter "single turn".

Let the initial lead angle of a helix $\alpha_{\boldsymbol{o}}$ be defined as the initial deformation of the spiral stent in the state without applied load.

The applied load on the spiral stent could be caused by the following physical quantities $F_{\boldsymbol{Z}}$ axial force, $F_{Z 1}$ axial force acting on the single turn, $F_{R}$ radial force, $F_{R \boldsymbol{I}}$ radial force acting on the single turn, $F_{T}$ tangential force, $p_{R}$ radial pressure.

The first step is to analyze the geometry of the spiral stent. The geometry of the stent is a structure of counter-rotating helix springs connected at their ends with a subsequent block uncoiling of the springs. Mutual dependence in this structure is between the length and diameter of the stent. The principle of the deformation of

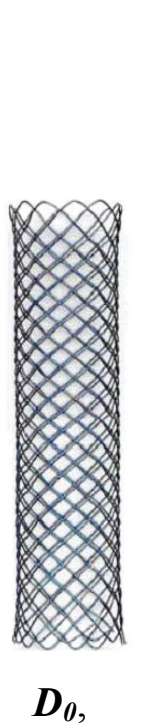

a)

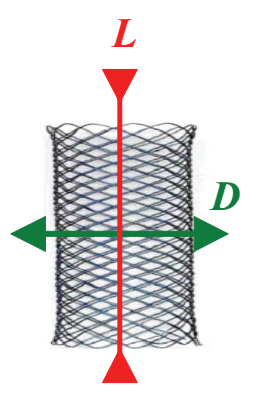

b)

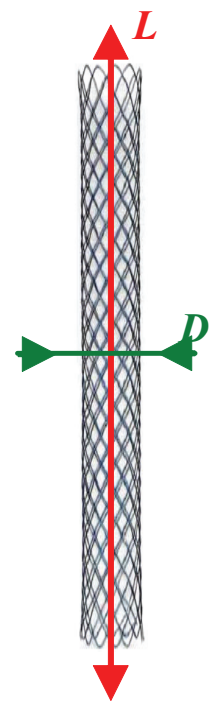

c)
Fig. 2. Schematics of the momenta in the load point $Q$.

the stent is shown in Fig. 1. It is clear now how to derive the corresponding equations describing the curvatures of the geometry of the wire of the spiral stent in dependence on the actual deformation of the spiral stent $\alpha$.

Obviously

$\xi=2 \pi \sin \alpha$

$\rho=2 \pi \cos \alpha$

The variables are $\xi$ as the angle of the bending of the wire of a single turn and $\varphi$ as the angle of the twisting of the wire of a single turn. Now we need to find out the principle of acting of the axial force $F_{Z}$ on the spiral stent. It is an important fact that $F_{\boldsymbol{Z}}$ acts at the edge of the stent - at the end-point of each coil of the stent, but not at the axis of the stent as shown in Fig. 2. This fact has a critical influence on the determination of the momentum $M$ of $F_{Z}$ and, in turn, on the final equation expressing the dependence of $F_{Z}$ on the stent deformation and the equation for transformation between $F_{Z}$ and $p$. We will use the analysis of the momentum $M$ of axial force $F_{Z 1}$.

We can see the relationships among the momenta of the axial force $F_{Z 1}$. It is clear now that

$M=M_{k} \cos \alpha-M_{o} \sin \alpha$

$|M|=2 r F_{Z 1}$

After substitution

$F_{Z I}=\left(M_{k} / 2 r\right) \cos \alpha-\left(M_{o} / 2 r\right) \sin \alpha$ 
Here $M_{k}$ is the twisting momentum and $M_{o}$ is the bending momentum of the axial force $F_{Z l}$. Now after substitution into Eq. (5) of the very well known equations describing each momentum

$M_{k}=\xi G J_{P} / l$

$M_{o}=\rho E J / l$

we will obtain Eq. (8)

$F_{Z l}=(\pi / l \cos \alpha)\left[\left(G J_{P} / l\right)\left(\xi-\xi_{o}\right) \cos \alpha+\left(E J / l\left(\rho-\rho_{o}\right) \sin \alpha\right]\right.$

The variables used are: $J_{\boldsymbol{P}}$ polar square momentum, $J$ square momentum of the cross-sectional area of the wire of the single turn; $\xi_{o}$ the initial angle of the bending of the wire of the single turn and $\varphi_{o}$ the initial angle of the twisting of the wire of the single turn; $E$ Young's modulus, $G$ shear modulus of the material of the wire of the single turn. Next we know that the whole stent has $n$ times $k$ of the single turn. After substitution of Eq. (1) and Eq. (2) into Eq. (8) we can derive and multiply the result by $n$ and $k$

$F_{Z}=\left(2 n k \pi^{2} / l^{2} \cos \alpha\right)\left[G J_{P}\left(\sin \alpha-\sin \alpha_{o}\right) \cos \alpha-E J(\cos \alpha-\right.$ $\left.\cos \alpha_{o}\right) \sin \alpha$ ]

Eq. (9) is the first result in our physical model describing the mutual dependence between the axial force $F_{\boldsymbol{Z}}$ and the deformation of the spiral stent $\alpha$.

The structure of the spiral stent allows us to derive easily the transformation equation between the axial force $F_{\boldsymbol{Z}}$ and the acting radial pressure $p_{\boldsymbol{R}}$ on the spiral stent. It is an important fact again that the radial pressure $p_{\boldsymbol{R}}$ and the forces causing this pressure act uniformly on the whole surface of the spiral stent. It means that the radial force $F_{R}$ and the tangential force $F_{T}$ are planar forces. We can simplify them for the model, as we neglect the diameter of the wire of the stent, to be curve forces. The derivation should be done as follows. It is valid that

$(\mathrm{d} z / 2) \mathrm{d} F_{T}=\mathrm{d} x F_{Z}$

It is clear from Fig. 3 that

$\mathrm{d} z / \mathrm{d} x=\tan \alpha=\sin \alpha / \cos \alpha$

After mutual substitution and integration we obtain

$F_{T}=2 F_{Z} \cos \alpha / \sin \alpha$

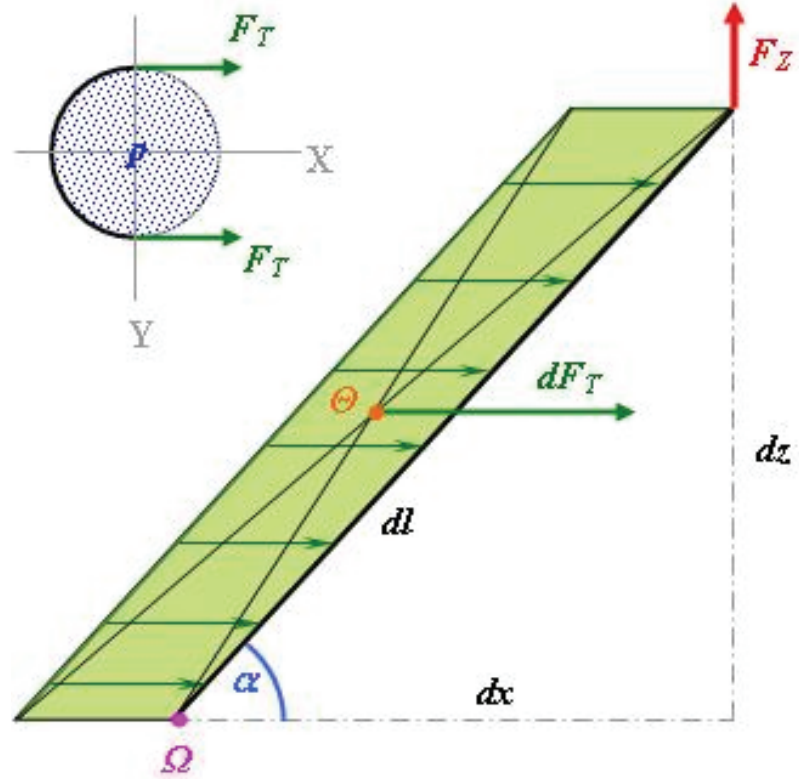

Fig. 3. Schematics showing the equilibrium state of the element of the single turn $d /$ between the element of the tangential force $\mathrm{d} F_{T}$ and the element of the axial force $\mathrm{d} F_{Z} . \Omega$ is the load point of the momenta of these forces. $\theta$ is the center of inertia of the curve force $\mathrm{d} F_{T}$.

Next we know that

$F_{T}=F_{R} / 2 \pi$

Figure 3 shows the situation.

It is easy to derive the following equation from the geometrical structure of the stent

$p_{R}=F_{R} / k l^{2}(\cos \alpha)(\sin \alpha)$

After substitution of Eq. 14 into Eq. 13 and then into Eq. 12 we obtain

$p_{R}=4 \pi F_{Z} / k l^{2} \sin ^{2} \alpha$

Eq. (15) is the second result in our physical model describing the mutual transformation between the axial force $F_{Z}$ and the radial pressure $p_{\boldsymbol{R}}$.

Eq. (15) is a very important equation. It allows us to perform the measurements of $F_{Z}$, because the measurements of the dependence of $F_{Z}$ on the stent deformation $\alpha$ are easy and not influenced by errors due to the measuring method (e.g. friction forces), in contrast with the measurements of $p_{R}$ or $F_{R}$. 


\section{$F_{Z^{--x}}$ dependence at $45^{\circ} \mathrm{C}$}

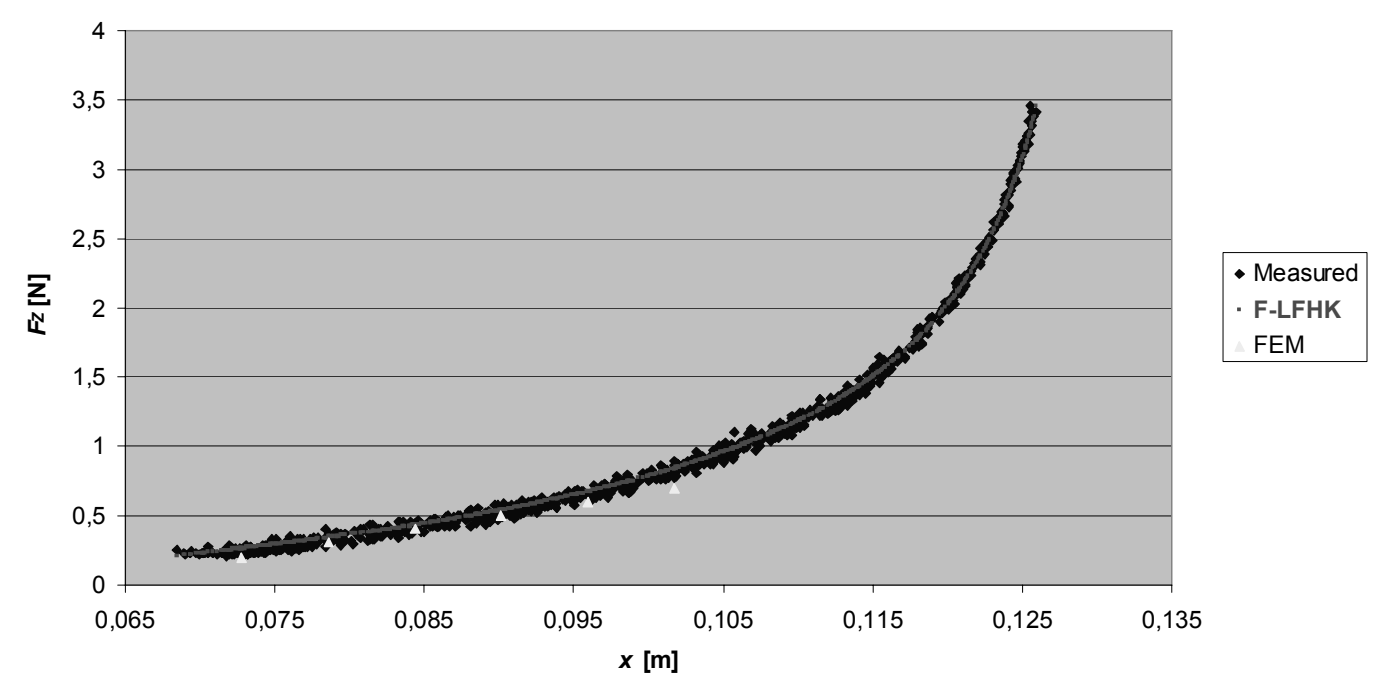

Fig. 4. Dependence of $F_{A}\left(F_{Z}\right)$ on the stent deformation; F-LFHK - our model, FEM - FEM model. This model was measured at $45{ }^{\circ} \mathrm{C}$ under very small load causing no transformation from austenite.

\section{Evidential analysis}

The Young modulus of treated Nitinol wire obtained from stress-strain tests had the approximate value for austenite is $60 \mathrm{GPa}$. The Young modulus of the stent material obtained by the least-squares method using our model is $62.69 \mathrm{GPa}$ (data measured at $45{ }^{\circ} \mathrm{C}$, austenite). The Young modulus of untreated Nitinol wire in austenite according to the approximate value given by the producer is $75 \mathrm{GPa}$. The measured parameters were: temperature $T=\left[{ }^{\circ} \mathrm{C}\right]$ with accuracy of $\pm 0.01{ }^{\circ} \mathrm{C} ; x=$ [mm] position of the free end of the stent with an accuracy of $\pm 0.200 \mathrm{~mm}$; axial force of the stent $F_{\boldsymbol{Z}}=[\mathrm{N}]$ with an accuracy of $\pm 0.030 \mathrm{~N}$. We measured all the input parameters of the stent needed for our mathematical model. Maximum limit value for our Newton meter "measuring head" was $5 \mathrm{~N}$. It can be seen that the data stated above and Fig. 4 clearly show the validity of our model.

\section{Application of FEM}

We used software COMSOL Multiphysics for FEM calculations. COMSOL is a complex environment including all the steps of modeling procedure: creating or importing the geometry, meshing the geometry, defining the physics on the domains and at the boundaries, solving the model, postprocessing the solution, and performing parametric studies.

FEM is very often used for modeling stents manufactured from metal tubing or stents formed from relatively simple repeating cells (Migliavica et al. 2005, Etave et al. 2001, Walke et al. 2005, Prendergast et al.
2003, Dumoulin et al. 2000).

The stent for which we developed an analytical model can be considered as a set of non-interacting spirals made from a very thin wire. So it is sufficient to work with a single spiral. Typically one coil is substituted with a series of straight beams connected together (Forrester 2001). Twisting and bending momentum is defined in the nodes (see Eq. 4 and Eq. 5) and the resulting set of equations (number corresponds with density of nodes) is solved by COMSOL. For small deformations it is sufficient to do only static stress analysis, the result is a linear relation between the axial force and deformation (Fig. 4). Results data may be extracted from the model: displacements, forces, stiffness, stress distribution and strains.

The virtual experiment was done by means of FEM. The problem was to investigate changes of mechanical properties of the spiral stent, if wires are welded where they are in contact. The simplified model consisting of two-one coil spirals was prepared. There was one "welded" joint. Using FEM the distribution of von Misses stress and strain energy was calculated. The strain energy of the "welded" sample was 1.3 times increased. For comparison of stress maps see Fig 5 .

\section{Discussion}

Both approaches have the application region where they are effective in spite of a lot of restrictions. The analytical model is a flexible tool for optimizing the delivery diameter in relation to desired stiffness for a 


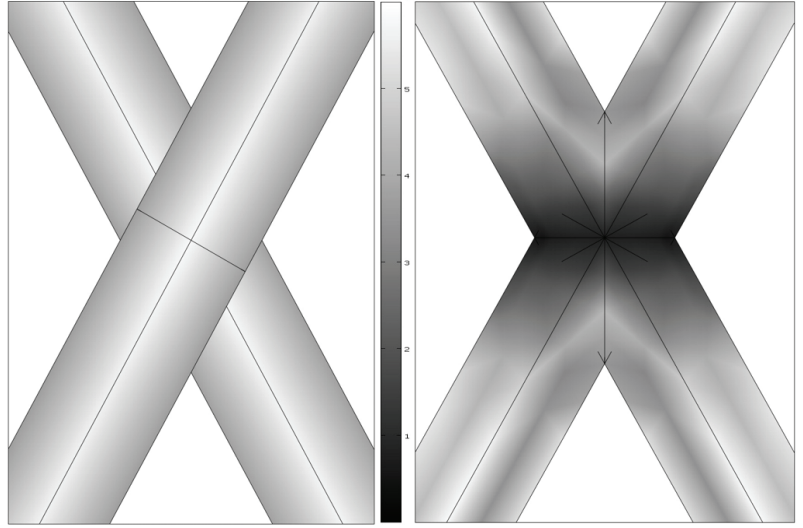

Fig. 5. Junction of two wires. Comparison of the von Misses stress distribution in "unwelded" (left) and "welded" (right) sample. The darker grey corresponds to smaller stress. The highest stress in the left sample is $0.6 \mathrm{GPa}$, in the rigtt sample it is $0.73 \mathrm{GPa}$. wire-woven spiral stent. But unfortunately it is practically impossible to apply it to more complicated shapes than the spiral. Our model also does not consider the fact that spirals are interwoven. The result of a contact between spirals is an additional bending of wires. It is important for large deformations. FEM is useful in "what if analyses" or it may be used for real-time simulations of manipulation with catheter or guide-wire (Lenoir et al. 2006) or it can help in preoperative choice of stent parameters by means of modifying its properties according to the real 3D image from MRA.

\section{Acknowledgements}

The project was funded by the Ministry of Industry and Trade, No.: FT-TA/097.

\section{References}

BEZROUK A, HANUS J, ZAHORA J: Temperature characteristics of nitinol spiral stents. Scripta Med (Brno) 78: 219-226, 2005.

DUMOULIN C, COCHELIN B: Mechanical behavior modeling of balloon-expandable stents. J Biomech 33: 1461$1470,2000$.

ETAVE F, FINET G, BOIVIN M, BOYER J C, RIOUFOL G, THOLLET G: Mechanical properties of coronary stents determined by using finite element analysis. J Biomech 34: 1065-1075, 2001.

HANUS J, ZAHORA J: Measurement and Comparison of Mechanical properties of Nitinol Stents, Phys Scripta 118: 264-267, 2005.

HOLZAPFEL GA, OGDEN RW: Mechanics of biological tissue. Springer-Verlag Berlin- Heidelberg, 2006, pp 225255.

LENOIR J, COTIN S, DURIEZ CH, NEUMANN P: Interactive physically-based simulation of catheter and guide-wire. Computers \& Graphics 30: 417-423, 2006.

KIM SM, PARK SY: Finite element analysis of stent expansion considering stent, artery and plaque interaction. In: International Multi-Conference Biomedical Engineering 2006 Insbruck, 2006, pp 143-146.

NING LI, YUANXIAN GU: Parametric design analysis and shape optimization of coronary arteries stent structure. In: World Congress of Structural and Multidisciplinary Optimization Rio de Janeiro, 30 May - 3 June 2005 , Brazil.

PENTRINI L, MIGLIAVACCA F, DUBINI G, AURICCHIO F: Evaluation of intravascular stent flexibility by means of numerical analysis. In: 2003 Summer Bioengineering Conference, June 25-29, Sonesta Beach Resort in Key Biscayne, Florida, 2003, pp 251-252.

PRENDERGAST PJ, LALLY C, DALY S, REID A, LEE TC, QUINN D, DOLAN F: Analysis of prolapse in cardiovascular stents: a constitutive equation for vascular tissue and finite-element modeling. J Biomech Engin 125: 692-699, 2003.

STOECKEL D, BONSIGNORE C, DUDA S: A survey of stent designs. Min Invas Ther \& Allied Technol 11: 137-147, 2002.

TAKASHIMA K, KITOU T, MORI K, IKEUCHI K: Simulation and experimental observation of contact conditions between stents and artery models. Med Engin Phys 29: 326-335, 2007.

WALKE W, PASZENDA Z, FILIPIAK J: Experimental and numerical biomechanical analysis of vascular stent. J Mater Process Tech 164-165: 1263-1268, 2005.

WANG R, RAVI-CHANDAR K: Mechanical response of a metallic aortic stent-part I: pressure-diameter relationship. J Appl Mech 71: 697-705, 2004. 
ZAHORA J, HANUS J: Design of nitinol stents. In: SMST-2004 Proceedings of International Conference on Shape Memory and Superelastic Technologies. M Mertmann (ed), 3-7 October, 2004 Baden-Baden, ASM International 2006, pp 171-176.

\section{Corresponding author}

Jiř́ Záhora, Department of Medical Biophysics, Faculty of Medicine, Charles University, Hradec Králové, Czech Republic. E-mail: zahora@1fhk.cuni.cz. 